\title{
Automatic Navigation System of Facility Agricultural Machinery Based on ZigBee
}

\author{
Changming Liu, ${ }^{1, a}$, Jie Tian ${ }^{1, b, *}$, Shi Luo ${ }^{2, c}$ and Ning Chen ${ }^{3, d}$ \\ ${ }^{1}$ College of Automobile and Traffic Engineering, Nanjing Forestry University, China \\ ${ }^{2}$ College of Automobile and Traffic Engineering, Jiangsu University, China \\ ${ }^{3}$ College of Mechanical and Electronic Engineering, Nanjing Forestry University, China \\ a1565654420@qq.com, bnjtianjie@163.com, cluoshi@ujs.edu.cn, ${ }^{d}$ chennning@njfu.com.cn
}

\begin{abstract}
Keywords: ZigBee; wireless networks; facility agricultural machinery; automatic navigation; tracking control
\end{abstract}

Abstract: Automatic navigation system of the facility agricultural machinery (FAM) is designed to realize the operation accuracy, production efficiency and using security, so that the farmers can be liberated from the heavy and monotonous field work. According to the indoor working characteristics of facility agricultures, a set of automatic navigation and positioning system based on ZigBee wireless communication mode is designed. The mathematical model of the FAM is established, and the path following fuzzy control strategy is proposed, whose inputs are the lateral deviation and the deviation of heading angle, and the output is the modification value of front wheel angle. Precise control of the steering motor is carried out. The hardware and software of the whole system are also disigned. Simulation and experiment of the system control strategy are carried out, and the results show that the final navigation and positioning accuracy of the ZigBee navigation system is around $0.2 \mathrm{~m}$ to $0.6 \mathrm{~m}$ and the system can meet the requirements of the FAM path tracking control system.

\section{Introduction}

The application of automatic navigation system make it possible for the facility agricultural machinery (FAM) to replace farmers in the field operations. In addition, it can also effectively increase the production efficiency, precision and operation safety of the agricultural machinery, reduce the labor costs and save the material resources and financial resources [1]. It is characterized in that the FAM with the automatic navigation system can get the surroundings information through the sensors installed on it, and make decisions by the navigation controller according to the target path given in advance and track the target path automatically. Considering that the facility agriculture belongs to the category of indoor positioning, it is necessary to establish a set of navigation system that can operate by itself because the GPS signal is unstable or can not be received in the facility agriculture environment [2]. At the same time, the navigation system should have several good characteristics such as lower power consumption, lower cost, smaller size and so on. Therefore the navigation system based on ZigBee is used [3]. What's more, the design, simulation and experimental study on the ZigBee navigation, path tracking control method and the vehicle steering control are carried on.

\section{Overall design of system}

In this paper, the intelligent FAM is based on the modified chassis of an electric vehicle. The automatic navigation system consists of a wireless network group formed by ZigBee and the modified electric vehicle chassis. Among them, the ZigBee wireless network group, which is composed of a base node group with known location information and a mobile node installed on the FAM with unknown location information of itself, is used to mearsure the location information of the mobile node. The modified electric vehicle chassis has a set of drive motor with a controlled area network (CAN) bus and its controller, and a steering-by-wire system in the type of worm gear and worm. Its working principle is to identify the vehicle motion attitude based on a variety of real time collected 
information, compute the target front-wheel steering angle according to the preset path information, send the motion control commands such as moving forward, backward or steering to the vehicle chassis and achieve the automatic driving of the facility agricultural vehicle.

\section{Hardware design of system}

In this paper two main control chips, STM32W108 of ST and MC9S12G48 of Freescale are used. The former is used to realize the ZigBee communication and the self-designed localization algorithm, and the latter is used to control the basic movement of the electric vehicle chassis, such as moving forward, drawing back and turning round.

In order to realize the automatic navigation, three types of ZigBee nodes have been set up. They are mobile node, base node and gateway node and their required hardware functions are a little different from each other. So it is necessary to have three kinds of hardware for ZigBee mobile node, ZigBee base node and ZigBee gateway node. All of them can implement ZigBee communication by STM32W108 integrated radio frequency. In addition, the mobile node hardware can also control the motion motor and the steering motor of the vehicle chassis, and the gateway node hardware has to achieve the serial communication of RS232 by the external serial port chip. Steering control is realized by setting up the control circuit of the DC motor. In the design, all the functions of the three nodes are designed into a single PCB as shown in Fig. 1. And it is necessary to weld the corresponding components according to the requirements of different nodes in actual usage.

In Fig.1, SAS angle sensor of BOSCH based on the Hall Effect is adopted. Its measuring range is $780^{\circ}$ and accuracy can reach $0.1^{\circ}$. According to its communication protocol, the angle of rotation can be obtained after setting the zero position. HMC6352 module of Honeywell, which integrates two mutually perpendicular magnetic resistance sensors and a digital circuit for directional computation, acts as the electronic compass sensor. The high accuracy heading information can be obtained by dealing with the Magnetic Field Strength. It is necessary to ensure the sensor directing to the North.

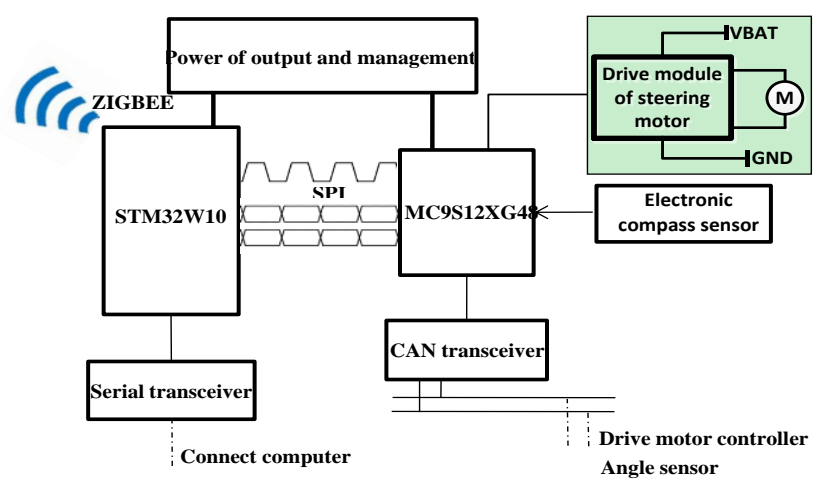

Fig.1 Function sketch map of PCB

\section{Systemic control strategy and algorithm}

Automatic navigation system mainly includes the navigation system based on ZigBee, path tracking system and steering actuator system. They are used to obtain the location and running state information of the vehicle, determine target angle of the FAM, and control the steering actuator motor to realize the FAM's automated driving according to the calculated target front wheel angle. The control block diagram is shown in Fig.2. 


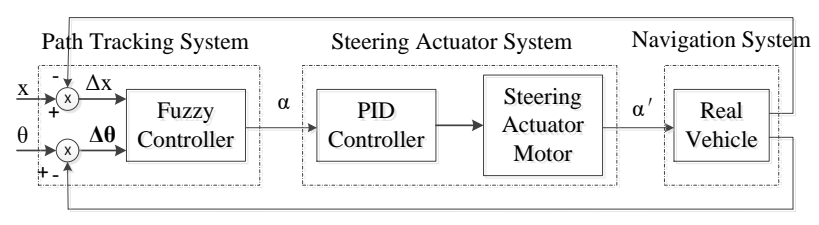

Fig.2 System control block diagram

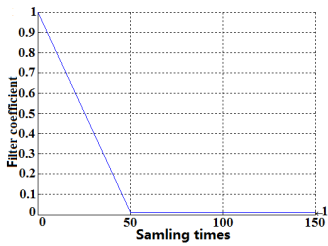

Fig.3 Table of filter coefficient

The ranging of Received Signal Strength Indicator (RSSI) based on ZigBee hardware is a ranging finding method by detecting the received signal strength value of two nodes (one is a fixed nodes, the other is a mobile node) in the process of communication, and putting the value mapped to the distance between the nodes. Considering the positioning will have some error caused by multipath interference, and non-visual factors [1], to this end the collected data require the following processing.

\section{Noise reduction processing}

In most cases the first-order filter with a lower filter coefficient has a poor sensitivity although its response stability is better. But when the initial value of the sampling is an abnormal point, the filter is very difficult to response to the subsequent sampling data. In fact, not only does the filter need to have good sensitivity at the early stages of the sampling but also it needs better response stability in the mid-late sampling. To solve the system error caused by the sampling initial value, a filter coefficient table is designed in advance (as shown in Fig.3) and the filter coefficients are dynamically set at each time.

In addition, the first-order filter system will have a larger error when the communication channel is continuously interfered by a barrier or under long time disturbance [4]. Obviously, the performance of the filter can be improved by the classification of the measured value mutation situation. The key problem is to determine the occurrence and the termination of RSSI mutation event. Based on the analysis of the real signal, the absolute value of the difference between the current value and the last value greater than or equal to a threshold (here set to 3 ) is regarded as the condition of the occurrence. And the current value back to near the RSSI value of the moment before the mutation occurrence (here set to \pm 1 ) is seen as the condition of the termination. Therefore, the signal processing according to the different events are as follows:

$$
\left\{\begin{array}{l}
Y(n)=Y(n-1)+K(X(n)-Y(n-1)), \text { at the occurence of RSSI mutation event. } \\
Y(n)=Y(n-1) \quad, \text { at the termination of RSSI mutation event }
\end{array} .\right.
$$

where $\mathrm{K}$ is the adaptive filter coefficient. When the mutation event occurs, $\mathrm{K}$ will be equal to its own multiplied by a coefficient greater than 1 . And this $\mathrm{K}$ value will be delayed for some time depended on the time of the measured value restored to the state when the mutation occurs.

\section{Dynamic distance forecast based on Kalman filtering}

If the distance and the observation variable between the mobile node and the base node are $\mathrm{d}$ and $\mathrm{d} \_\mathrm{m}$, the system equation and observation equation are as follows:

$$
\left\{\begin{array}{l}
d(k)=E \cdot d(k-1)+w(k) \\
d \_m(k)=H \cdot d(k)+v(k)
\end{array}\right. \text {. }
$$

where $\mathrm{E}$ is one-step transition matrix of $\mathrm{d}$, which can be approximated to 1 due to the low speed of the agricultural machinery. $\mathrm{H}$ is the observation system parameter, which indicates the gain of the observed variable affected by the state variable and the value is $1 . \mathrm{w}(\mathrm{k})$ and $\mathrm{v}(\mathrm{k})$ are the white noises which are independent of each other and fit the normal distribution. 
If $\mathrm{d}(\mathrm{k}-1 \mid \mathrm{k}-1)$ is the distance between mobile node and base node at the time of $\mathrm{k}-1$, the distance of the current moment $\mathrm{k}$ can be expressed as $\mathrm{d}(\mathrm{k} \mid \mathrm{k}-1)=\mathrm{Ed}(\mathrm{k}-1 \mid \mathrm{k}-1)$. If $\mathrm{P}_{\mathrm{d}}(\mathrm{k}-1 \mid \mathrm{k}-1)$ is the covariance of $\mathrm{d}(\mathrm{k} \mid \mathrm{k}-1)$, then $\mathrm{P}_{\mathrm{d}}(\mathrm{k}-1 \mid \mathrm{k}-1)=\mathrm{P}_{\mathrm{d}}(\mathrm{k} \mid \mathrm{k}-1)+\mathrm{Q}$. And the Kalman gain of the current time is as follows:

$$
K_{d}(k)=\frac{P_{d}(k \mid k-1)}{P_{d}(k \mid k-1)+R}
$$

where $\mathrm{R}$ is the measurement noise covariance of $\mathrm{v}(\mathrm{k})$.

Then the optimal estimate of the current state $\mathrm{d}(\mathrm{k} \mid \mathrm{k})$ and its covariance $\mathrm{P}_{\mathrm{d}}(\mathrm{k} \mid \mathrm{k})$ are as follows:

$$
\left\{\begin{array}{l}
\mathrm{d}(\mathrm{k} \mid \mathrm{k})=\mathrm{d}(\mathrm{k} \mid \mathrm{k}-1)+\mathrm{K}_{\mathrm{d}}(\mathrm{k})\left(\mathrm{d} \_\mathrm{m}(\mathrm{k})-\mathrm{d}(\mathrm{k}-1 \mid \mathrm{k}-1)\right. \\
\mathrm{P}_{\mathrm{d}}(\mathrm{k} \mid \mathrm{k})=\left(1-\mathrm{K}_{\mathrm{d}}(\mathrm{k})\right) \mathrm{P}_{\mathrm{d}}(\mathrm{k} \mid \mathrm{k}-1)
\end{array} .\right.
$$

\section{Path tracking controller}

Assuming that the FAM is drivered by the rear wheels and steered by the front wheels, the kinematics equation with the vehicle's rear axle as center point can be set up as Eq.5 [5], where $\mathrm{x}$ and $\mathrm{y}$ are the coordinates of vehicle mass center point $(\mathrm{m}), \theta$ is vehicle yawing angle $(\mathrm{rad}), \mathrm{v}$ is vehicle speed $(\mathrm{m} / \mathrm{s})$, $\mathrm{u}$ is steering angular velocity $(\mathrm{rad} / \mathrm{s}), \alpha$ is front wheel Angle ( $\mathrm{rad})$ and L is the wheelbase of the vehicle (m).

$$
\left\{\begin{array}{l}
\dot{\mathrm{x}}=\mathrm{v} \sin \theta \\
\dot{\mathrm{y}}=\mathrm{v} \cos \theta \\
\dot{\theta}=\frac{\mathrm{v}}{\mathrm{L}} \tan \alpha \\
\dot{\alpha}=\mathrm{u}
\end{array} .\right.
$$

Because the FAM's work path is usually composed of multiple linear line path, rather than any curve paths. As a result, we only consider the heading tracking caused by itself structure or road surface of the facility agriculture. In this paper, a fuzzy controller with double input (deviation of heading angle $\Delta \theta$, lateral deviation $\Delta \mathrm{x}$ ) and single output (correction of the front wheel angle $\Delta a$ ) are designed to control the vehicle lateral deviation

Here 7 language variables are chosen to describe the input and output variables in the fuzzy control, which are Plus-Big (PB), Plus-Middle(PN), Plus-Small (PS), Zero (ZE) ,Negative-Small(NS), Negative-Middle (NM) and Negative-Big(NB). The basic domain of $\Delta \mathrm{x}, \Delta \theta$ and $\Delta \mathrm{a}$ are [0.8 $0.8 \mathrm{~m}$ ], $\left[-10^{\circ} 10^{\circ}\right]$ and $\left[-10^{\circ} 10^{\circ}\right]$ respectively. When the vehicle locates on the left side of the planned path, $\Delta \mathrm{x}$ is positive. The clockwise variable of $\Delta \theta$ and $\Delta a$ are positive. In order to calculate conveniently, triangular membership function are chosed as the membership function. The membership function curves of $\Delta \theta, \Delta \mathrm{x}$ and $\Delta \mathrm{a}$ are shown in Fig.4. According to the relative position between the vehicle and the planning path, a total of 49 control rules were established, and the fuzzy control rule table is shown in Table 1.

The vehicle speed and the heading angle under the planned path are set to $0.5 \mathrm{~m} / \mathrm{s}$ and $0^{\circ}$ respectively. The simulation results of six groups $\left(\Delta \mathrm{x}=0.8 \mathrm{~m}, \Delta \theta= \pm 10^{\circ} ; \Delta \mathrm{x}=0 \mathrm{~m}, \Delta \theta= \pm 10^{\circ}\right.$; $\Delta x=-0.8 \mathrm{~m}, \Delta \theta= \pm 10^{\circ}$ ) are shown in Fig.5. As shown in Fig.5, all of the error between the vehicle and planned path can be reduced by adjusting the front wheel angle, which proves that the fuzzy controller has good tracking performance. 


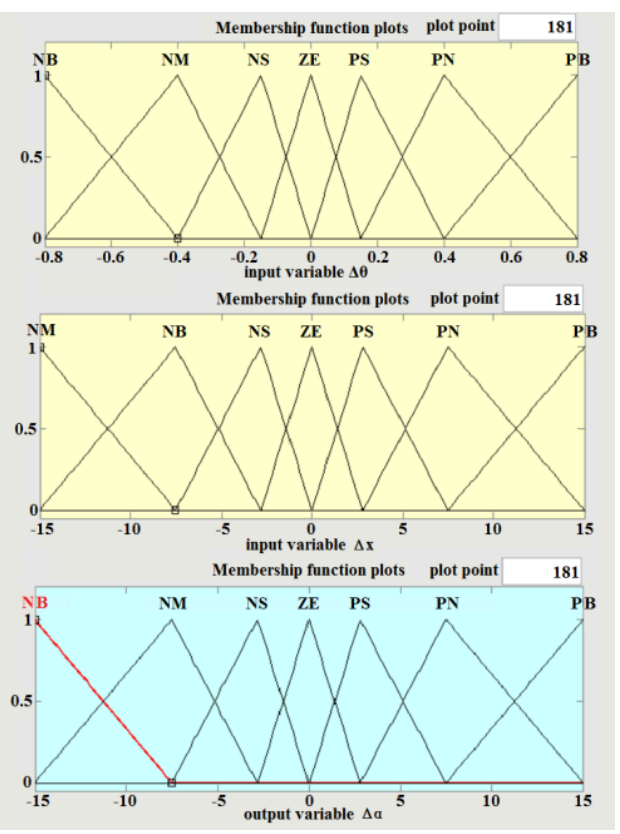

Fig.4 Curves of membership functions
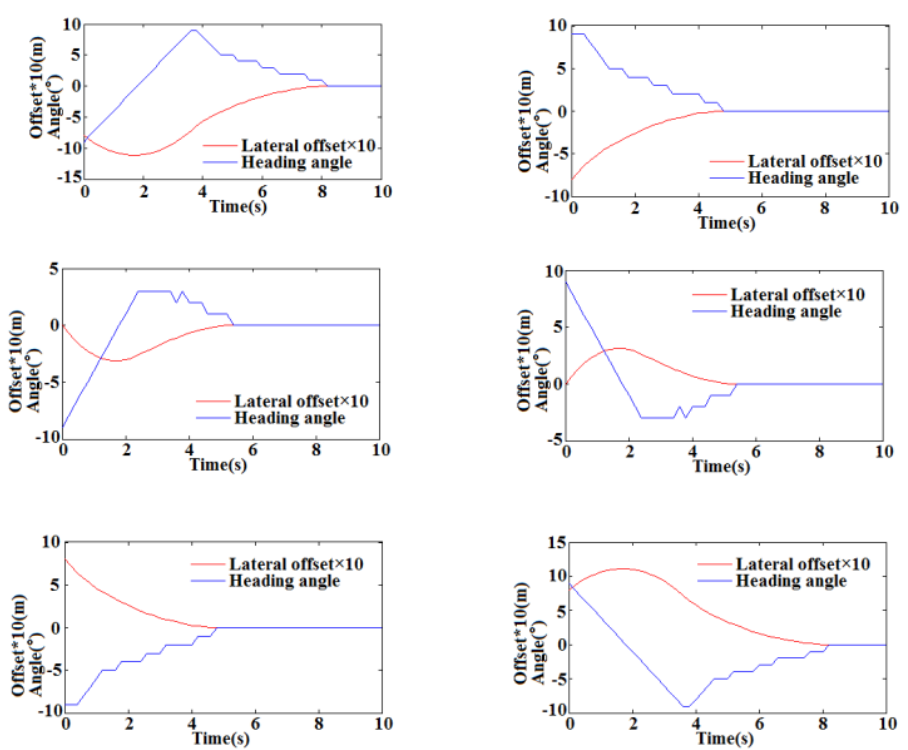

Fig.5 Simulation results of heading tracking

Table 1 Fuzzy control rule table

\begin{tabular}{|c|c|c|c|c|c|c|c|}
\hline$\Delta \theta$ & $N B$ & $N M$ & $N S$ & $Z E$ & $P S$ & $P M$ & $P B$ \\
\hline NB & PB & PB & PB & PM & PS & PS & NB \\
\hline NM & PB & PB & PM & PS & PS & ZE & NS \\
\hline NS & PB & PM & PS & PS & ZO & NS & NM \\
\hline ZE & PM & PM & PS & ZE & NS & NM & NM \\
\hline PS & PM & PS & ZE & NS & NS & NM & NB \\
\hline PM & PS & ZE & NS & NS & NM & NB & NB \\
\hline PB & ZE & NS & NS & NM & NM & NB & NB \\
\hline
\end{tabular}

\section{PID control of steering actuator system}

For a vehicle, the function of path tracking is ultimately realized by the steering actuator system. Here the PID parameters are adjusted firstly on the steer-by-wire test bench. Considering that the continuous PID controller cannot be directly applied to the computer control system [6], so it need to be turned into the discrete PID controller and the formula is as follows:

$$
\mathrm{U}(\mathrm{k})=\mathrm{k}_{\mathrm{p}}\left(\mathrm{e}(\mathrm{k})+\frac{\mathrm{T}}{\mathrm{T}_{\mathrm{t}}} \sum_{\mathrm{j}=0}^{\mathrm{K}} \mathrm{e}(\mathrm{j})+\frac{\mathrm{T}}{\mathrm{T}_{\mathrm{D}}}[\mathrm{e}(\mathrm{k})-\mathrm{e}(\mathrm{k}-\mathrm{l})]\right)
$$

where $\mathrm{k}$ is the sampling number, $\mathrm{U}(\mathrm{k})$ is the output value at the time $\mathrm{k}, \mathrm{e}(\mathrm{k})$ and $\mathrm{e}(\mathrm{k}-1)$ are the input deviations at the sampling time $\mathrm{k}$ and $\mathrm{k}-1$ respectively, $\mathrm{T}$ is sampling period.

Fig. 6 is the curve of the steering following performance for the test bench, which proves that the PID controller has good steering following performance and it is feasible to modify to the vehicle. 


\section{Real vehicle test}

The electric vehicle chassis used in the experiment is shown in Fig.7. The electronic compass sensor and mobile node controller are fixed on the vehicle. And the mobile node controller is connected with the drive-motor controller and angle sensor by CAN bus, and with the steering motor and electronic compass sensor by the hardware.

According to the size of the actual site, $30 \mathrm{~m} \times 20 \mathrm{~m}$ area composed of 12 base node sensors is chosen, a planned path of L type is set. When base node coordinates and planning path are configured in the automatic navigation software and the path loss model is calibrated on the PC, then switch to the monitoring interface of driving path, and the experiment can be carried out after setting the vehicle speed as $1 \mathrm{~m} / \mathrm{s}$ and the sampling period as $1000 \mathrm{~ms}$,

In the experiment the whole running process of the vehicle is shown in Fig.8, where the red line and the black one are the planned path and the actual localization curve respectively. It can be drawn that the output of the vehicle navigation system is basically consistent with the actual path, which proves the system is reliable. And the location accuracy of the navigation control system based on ZigBee is $0.2-0.6 \mathrm{~m}$ by analysing the sampling data through the software.

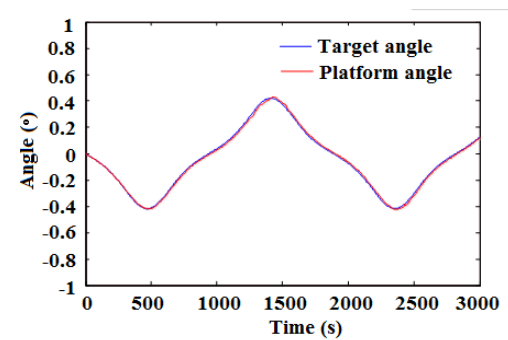

Fig.6 Steering performance test

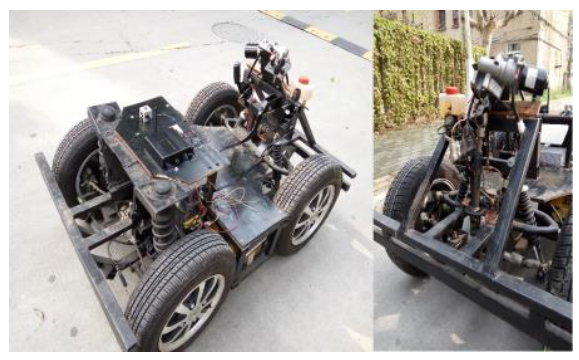

Fig.7 vehicle chassis for test

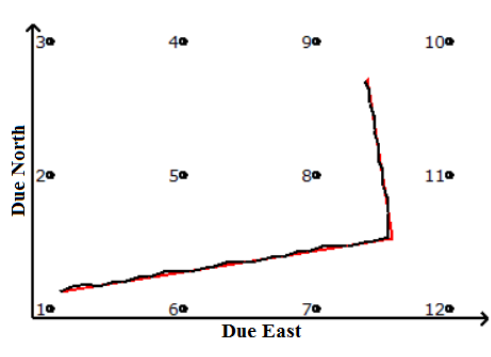

Fig.8 Path curves

\section{Conclusion}

In this paper, the automatic navigation system of facility agricultural machinery which consists of the navigation system based on Zigbee, path tracking system and steering actuator system is designed independently. The simulation and experiment show that the system can effectively achieve the automatic navigation function of the facility agricultural machinery.

\section{Acknowledgements}

This work was supported by National Natural Science Foundation of China (11272159), and National Natural Science Foundation for youth of China (51305207), Jiangsu Government Scholarship for Overseas Studies (JS-2014-014) and Technological Innovation Fund Project of Nanjing Forestry University (163106007).

\section{References}

[1] T.J.Gao, J.G Ma: submitted to Xinjiang Agricultural Mechanization (2014) (In Chinese).

[2] J.T.Hu, L.Gao, X.P.Bai, et al: Transactions of the Chinese Society of Agricultural Engineering 2015 (In Chinese).

[3] G.B.Xiao, J.Tian, J.H.Zhong: submitted to Control \& Automation (2010) (In Chinese).

[4] Y.Ji, Z.X.Liu, G.Liu, et al: submitted to Transactions of the Chinese Society for Agricultural Machinery (2009) (In Chinese).

[5] Z.X.Liu, G.Liu, Y.Ji, et al: submitted to Transactions of the Chinese Society for Agricultural Machinery (2010) (In Chinese). 
[6] J.Tian, G X.ao, N.Chen, et al: submitted to Journal of Machine Design (2011) (In Chinese). 